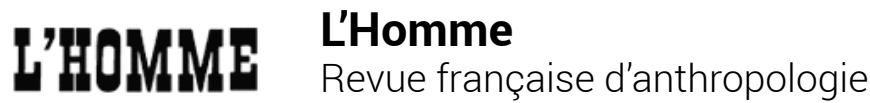

187-188 | 2008

Miroirs transatlantiques

\section{Francine Hirsch, Empire of Nations. Ethnographic Knowledge and the Making of the Soviet Union}

\section{Frédéric Bertrand}

\section{(2) OpenEdition \\ 9 Journals}

\section{Édition électronique}

URL : https://journals.openedition.org/lhomme/21032

DOI : 10.4000/lhomme.21032

ISSN : 1953-8103

Éditeur

Éditions de l'EHESS

\section{Édition imprimée}

Date de publication : 3 octobre 2008

Pagination : 533-534

ISBN : 978-2-7132-2186-6

ISSN : 0439-4216

\section{Référence électronique}

Frédéric Bertrand, «Francine Hirsch, Empire of Nations. Ethnographic Knowledge and the Making of the Soviet Union ", L'Homme [En ligne], 187-188 | 2008, mis en ligne le 16 décembre 2008, consulté le 24 avril 2022. URL : http://journals.openedition.org/lhomme/21032 ; DOI : https://doi.org/10.4000/ Ihomme.21032

Ce document a été généré automatiquement le 24 avril 2022.

(c) École des hautes études en sciences sociales 


\title{
Francine Hirsch, Empire of Nations. Ethnographic Knowledge and the Making of the Soviet Union
}

\author{
Frédéric Bertrand
}

\section{RÉFÉRENCE}

Francine Hirsch, Empire of Nations. Ethnographic Knowledge and the Making of the Soviet

Union. Ithaca-London, Cornell University Press, 2005, 367 p., bibl., index, ill., cartes.

1 L'OUVRAGE DE l'historienne Francine Hirsch, publié dans la collection «Culture and Society after Socialism» dirigée par deux anthropologues spécialistes de la Russie, Bruce Grant et Nancy Ries, reprend les principaux axes de la thèse qu'elle a soutenue en 1998 à l'Université de Princeton, en y incluant de nouvelles perspectives, notamment comparatives.

2 L'objectif de l'auteure est de mettre au jour l'implication de l'anthropologie soviétique des années 1920-1930 dans la formation de l'unss. Pour se faire, Francine Hirsch s'appuie sur un groupe d'experts, des anthropologues membres des deux institutions phares dans le domaine : la Commission d'étude de la composition tribale de la Russie et des pays limitrophes et le département d'ethnographie du Musée russe, tous deux situés à Leningrad. Par l'analyse minutieuse de ce qu'elle nomme une "technologie coloniale ", c'est-à-dire le recensement, la cartographie et les expositions ethnographiques, Francine Hirsch rend visibles quelques-unes des étapes essentielles du travail de négociation mené conjointement par les anthropologues et les différents acteurs du projet de construction de la société soviétique stalinienne.

3 Manifestement inspirée par les travaux de G. W. Stocking Jr sur l'anthropologie britannique et nord-américaine, Francine Hirsch porte toute son attention sur les conditions d'objectivation du principal domaine d'expertise de l'anthropologie soviétique naissante: l'intégration de l'ethnicité dans la construction de l'identité 
soviétique. Si le recours à la cartographie et au recensement dans le contexte soviétique a déjà fait l'objet de nombreuses études, le musée ethnographique en tant que laboratoire reste encore largement inexploré. Y consacrant son cinquième chapitre, l'auteure s'attache tout particulièrement à rappeler la progressive construction d'une communauté de langage née des pratiques de négociation entre les intérêts et les attentes des administrations, du public et des anthropologues, et notamment l'extraordinaire montée en puissance d'un régime de scientificité fonctionnant très largement sur le principe de l'échantillonnage et de la preuve par l'image'.

4 L'atout majeur de cette étude repose sur le soin apporté par Francine Hirsch à ne rien masquer de l'élaboration d'un véritable compromis sur les conditions acceptables d'application des catégories et des savoir-faire anthropologiques. Dès lors, sous l'argument martelé d'un utilitarisme volontariste affiché dans les discours et les slogans pointe la mise en contexte d'une instrumentalisation réciproque. On devrait savoir assez gré à l'auteure de rappeler par là même que le compromis n'est pas pour autant synonyme de compromission. Hormis la mention faite de quelques actes de résistance et stratégies d'évitement mis au crédit des anthropologues, on ne prend cependant que trop peu conscience que leur volonté de pérenniser institutionnellement leur discipline en Russie soviétique s'est révélée en somme une véritable rencontre manquée. En effet, durant les purges staliniennes, les anthropologues ont vu à de multiples reprises se retourner contre eux les principes mêmes de leur légitimité et de leur implication : connaissance de la langue du groupe étudié, séjours réguliers et prolongés sur place, position de porte-parole qui les désignaient coupables dans plusieurs simulacres de procès pour espionnage en faveur notamment de la Finlande et du Japon.

5 À ce titre, s'il convient de remarquer que Francine Hirsch a su parfaitement éviter les pièges de la martyrologie officielle ${ }^{2}$, il n'en reste pas moins déconcertant de noter une certaine digression dans l'argumentation. En effet, la troisième et dernière partie consacrée à la Terreur stalinienne et à la mise en parallèle des débats en URSS et dans l'Allemagne nazie autour de la classification ethnique, même si elle se justifie dans le contexte historique étudié, tend néanmoins à faire tomber la représentation de l'anthropologie soviétique proposée jusque-là dans l'ornière de la dichotomie science normale/science idéologisée. Or, sans nier l'importance et la réalité du traumatisme de la «marxisation", il me semble important de soutenir les efforts de nombreux anthropologues russes pour comprendre et faire comprendre l'anthropologie des années 1930 selon sa logique et ses termes propres. De plus, au lieu d'une mise en parallèle avec la Volkskunde nazie, il y a fort à parier que notre compréhension de l'anthropologie soviétique aurait gagné autant, sinon plus, à la comparaison des mécanismes de réponse à la commande sociale mis en place à la même période par une autre anthropologie nationale, elle-même en plein contexte de légitimation. Et cela d'autant plus que les relations avec l'ensemble des grandes traditions nationales en anthropologie s'avéraient encore bien réelles.

6 Cette remarque faite, il reste que l'analyse scrupuleuse des sources, le souci du détail, la prise en compte de la complexité des processus de légitimation en jeu font de cet ouvrage un outil précieux pour une réflexion nécessaire quant à la possibilité de faire de l'anthropologie russe et soviétique un véritable objet d'étude anthropologique. 


\section{NOTES}

1. Frédéric Bertrand, «Des expositions de papiers : musée et objet ethnographiques dans l'URSS des années 1930 », Gradhiva, 2003, 34 : 29-37.

2. Nathaniel Knight, " "Salvage Biography" and Useable Pasts : Russian Ethnographers Confront the Legacy of Terror ", Kritika : Explorations in Russian and Eurasian History, 2000, 2 (1): 365-376. 